\title{
EXISTENCE OF A STRONG LIFTING COMMUTING WITH A COMPACT GROUP OF TRANSFORMATIONS II
}

\author{
RUSSELL A. JoHNSON
}

\begin{abstract}
Let $G$ be a locally compact group with left Haar measure $\gamma$. The well-known "Theorem LCG" of A. and C. IonescuTulcea states that there is a strong lifting of $M^{\infty}(G, \gamma)$ commuting with left translations. Our purpose here is to prove a generalization of this theorem in case $G$ is compact. Thus let $(G, X)$ be a free left transformation group with $X$ and $G$ compact. Let $\nu_{0}$ be a Radon measure on $Y=X / G$, and let $\mu$ be the Haar lift of $\nu_{0}$. Let $\rho_{0}$ be a strong lifting of $M^{\infty}\left(Y, \nu_{0}\right)$. We will show that $M^{\infty}(X, \mu)$ admits a strong lifting $\rho$ which extends $\rho_{0}$ and commutes with $G$.
\end{abstract}

In [6], the result just stated was proved when $G$ and $X$ satisfied certain restrictions. The following theorem, which may be of independent interest, enables us to remove the conditions imposed in [6]: Let $H$ be a closed normal Lie subgroup of a compact group $G$ : then there is a $D^{\prime}$ sequence (see 1.2 and [1] in $H$, consisting of compact neighborhoods $V_{n}(n \geqq 1)$ of the identity, such that $g^{-1} V_{n} g=V_{n}$ for all $g \in G$.

1.

Notation 1.1. Let $G$ be a compact topological group, $H$ a closed, normal, real Lie subgroup. Let $\gamma$ be normalized Haar measure on $G$, and let $\lambda$ be normalized Haar measure on $H$. For each $g \in G$, define $\alpha_{g}: H \rightarrow H: h \rightarrow g^{-1} h g$. Let $\mathfrak{S}$ be the Lie algebra of $H$; let exp: $\widetilde{\mathfrak{C}} \rightarrow H$ be the exponential map.

Definition 1.2. ([1]). A $D^{\prime}$-sequence in $H$ is a sequence $\left(W_{n}\right)_{n=1}^{\infty}$ of $\lambda$-measurable subsets of $H$ such that (i) $W_{n} \supset W_{n+1}(n \geqq$ 1); (ii) $0<\lambda\left(W_{n} W_{n}^{-1}\right)<C \cdot \lambda\left(W_{n}\right)$ for some $C>0$ and all $n$; (iii) every neighborhood of idy ( $\equiv$ identity) $\in H$ contains some $W_{n}$.

Proposition 1.3. There is a $D^{\prime}$-sequence $\left(V_{n}\right)_{n=1}^{\infty}$ in $H$, consisting of compact neighborhoods of idy, such that $g^{-1} V_{n} g=V_{n}(n \geqq 1$, $g \in G)$.

Proof. Let $W$ be a neighborhood of 0 in $\mathfrak{F}$ such that $\exp \mid w$ is a diffeomorphism onto $\exp (W) \subset H_{0}$, the identity component of $H$. Define $\log$ to be the inverse of $\left.\exp \right|_{W}$. There is a neighborhood $N \subset \exp (W)$ of idy such that $g^{-1} N g \subset W(g \in G)$. Let $\varphi_{g}(x)=\log \circ \alpha_{g} \circ$ 
$\exp (x)=\log \left(g^{-1} \cdot \exp (x) \cdot g\right)$ for all $x \in W_{1}=\log (N)$. Then $\varphi_{g}: W_{1} \rightarrow$ $W$, and $\varphi_{g}(0)=0(g \in G)$.

Each $\operatorname{map} \alpha_{g}$ is a continuous isomorphism of $H$, hence is analytic ([9], Theorem 5.22). Let $\operatorname{Ad}_{g}: \mathfrak{F} \rightarrow \mathfrak{S}$ be the derivative at idy $\in H$ of $\alpha_{g}$. Then $\operatorname{Ad}_{g}(x)=D \varphi_{g}(0) \cdot x(x \in \mathfrak{S})$. The map $g \rightarrow \operatorname{Ad}_{g}$ is a homomorphism of $G$ into $G L(\mathfrak{K})$. We show that it is continuous. Let $G_{0}=\left\{g \in G \mid g^{-1} h g=h\right.$ for all $\left.h \in H_{0}\right\}$. Then $G_{0}$ is a closed normal subgroup of $G$. The group $G / G_{0}$ acts effectively on $H_{0}$ via the map $\eta: G / G_{0} \times H_{0} \rightarrow H_{0}:\left(g G_{0}, h\right) \rightarrow g^{-1} h g$. Therefore $G / G_{0}$ is a Lie group, and the map $\eta$ is analytic ([8], pp. 208, 212, 213). It follows that $g \rightarrow \mathrm{Ad}_{g}$ is continuous.

Let $\langle,\rangle_{1}$ be an inner product on $\mathfrak{S}$. Define an inner product $\langle$,$\rangle , invariant under each \mathrm{Ad}_{g}$, by

$$
\langle x, y\rangle=\int_{G}\left\langle\operatorname{Ad}_{g}(x), \operatorname{Ad}_{g}(y)\right\rangle_{1} d \gamma(g)(x, y \in \mathfrak{S}) \text {. }
$$

Observe that, if $B_{r}=\left\{x \in \mathfrak{S} \mid\|x\| \leqq r\right.$, where $\left.\|x\|^{2}=\langle x, x\rangle\right\}$, then $\operatorname{Ad}_{g}\left(B_{r}\right)=B_{r}(g \in G)$. Also observe that, if $m$ is a Lebesgue measure on $\mathfrak{K}$, then there is a constant $\beta$ such that $m\left(B_{r}\right)=\beta r^{k}$, where $k=$ $\operatorname{dim} H$.

Consider the measure $\left.\lambda\right|_{\exp W}$. By ([7], Corollary 2, p. 106), there is a Lebesgue measure $m$ on $\mathfrak{S}$ and an analytic function $\rho: W \rightarrow \boldsymbol{R}$, satisfying $\rho(0)=1$, such that $\lambda(\exp B)=\int_{B} \rho(x) d m(x)$ for each Borel set $B \subset W$. Let $W_{2}$ be a neighborhood of $0 \in \mathfrak{F}$ such that $1 / 2 \leqq$ $\rho(x) \leqq 2\left(x \in W_{2}\right)$.

Now let $0<\varepsilon<1$ satisfy $(1-\varepsilon)^{k}>1 / 2(k=\operatorname{dim} H)$. Recall that $\varphi_{g}(0)=0$ for all $g \in G$, that $\operatorname{Ad}_{g}(x)=D \varphi_{g}(0) \cdot x$, that $G$ is compact, and that $\left(g G_{0}, x\right) \rightarrow \varphi_{g}(x): G / G_{0} \times W_{2} \rightarrow W$ is analytic. We can therefore find $r^{\prime}>0$ such that

$\left(^{*}\right)\left\|\varphi_{g}(x)-\operatorname{Ad}_{g}(x)\right\|<\varepsilon\|x\|$ for all $g \in G$ if $\|x\| \leqq r^{\prime}$ (recall $\left.\|x\|^{2}=\langle x, x\rangle\right)$. Choose $r_{0} \leqq r^{\prime}$ such that $B_{3 r} \subset W_{2}$ and $\exp \left(B_{r}\right) \cdot$ $\exp \left(B_{r}\right) \subset \exp B_{3 r}$ if $r \leqq r_{0}$. Let $r_{n}=r_{0} / n$. Define $C_{n}=\bigcap_{g \in G} \Phi_{g}\left(B_{r_{n}}\right)$, and let $V_{n}=\exp \left(C_{n}\right)$. By $\left({ }^{*}\right), B_{(1-\varepsilon) r_{n}} \subset C_{n}$ for each $n$. Hence $V_{n}$ is a compact neighborhood of idy for each $n(n \geqq 1)$.

We show that $\left(V_{n}\right)_{n=1}^{\infty}$ is the desired $D^{\prime}$-sequence in $H$. First note that $g^{-1} V_{n} g=\alpha_{g} \circ \exp \left(C_{n}\right)=\exp \circ \varphi_{g}\left(C_{n}\right)=\exp C_{n}=V_{n}$ for all $g \in G$. Next, observe that $V_{n} V_{n}^{-1}=\exp \left(C_{n}\right) \cdot \exp \left(-C_{n}\right) \subset \exp \left(B_{r_{n}}\right) \cdot \exp \left(B_{r_{n}}\right) \subset \exp B_{3 r_{n}}$. So $\exp \left(B_{(1-\varepsilon) r_{n}}\right) \subset V_{n} \subset V_{n} V_{n}^{-1} \subset \exp B_{3 r_{n}}$. So, on the one hand, $\lambda\left(V_{n} V_{n}^{-1}\right) \leqq$ $\lambda\left(\exp B_{3 r_{n}}\right)=\int_{B_{3 r_{n}}} \rho(x) d m(x) \leqq 2 \cdot \beta \cdot 3^{k} \cdot\left(r_{n}\right)^{k}$, while on the other hand,

$$
\lambda\left(V_{n}\right) \geqq \int_{B_{(1-\varepsilon) r_{n}}} \rho(x) d m(x) \geqq 1 / 2 \beta(1-\varepsilon)^{k}\left(r_{n}\right)^{k}>1 / 4 \beta\left(r_{n}\right)^{k} .
$$

Hence $\lambda\left(V_{n} V_{n}^{-1}\right) \leqq 8 \cdot 3^{k} \lambda\left(V_{n}\right)$, so (ii) of 1.2 is satisfied with $C=8 \cdot 3^{k}$. 
It is easy to see that $\left(V_{n}\right)_{n=1}^{\infty}$ satisfies (i) and (iii) of 1.2. This completes the proof of 1.3 .

REMARK 1.4. The sequence $\left(V_{n}\right)_{n=1}^{\infty}$ is also a $D^{\prime \prime}$-sequence ([1]); that is, each $V_{n}$ contains a subset $U_{n}$ such that $U_{n} \cup U_{n} U_{n}^{-1} \subset V_{n}$, and $\lambda\left(V_{n}\right)<C^{\prime} \lambda\left(U_{n}\right)$ for some constant $C^{\prime}(n \geqq 1)$. To see this, let $s_{n}=(1-\varepsilon) r_{n} / 3$, and let $U_{n}=\exp B_{s_{n}}$. Then $U_{n} \cdot U_{n}^{-1} \subset \exp B_{(1-s) r_{n}} \subset$ $V_{n}$, and it is easy to see that we may choose $C^{\prime}=8 \cdot 3^{k}$.

2. The reader is warned that much of the terminology of this section was discussed in ([6]); that discussion will not be repeated in all detail.

Notation 2.1. Let $X$ be a compact Hausdorff space, and let $G$ be a compact Hausdorff topological group. Suppose $(G, X)$ is a (left) transformation group (thus there is a continuous map $\Phi: G \times$ $X \rightarrow X:(g, x) \rightarrow g \cdot x$ satisfying (i) idy $\cdot x=x$; (ii) $g_{1} \cdot\left(g_{2} \cdot x\right)=\left(g_{1} g_{2}\right) \cdot$ $\left.x\left(x \in X ; g, g_{1}, g_{2} \in G\right)\right)$. Suppose also that $G$ acts freely (thus $g \cdot x=$ $x \Rightarrow g=\operatorname{idy}(g \in G, x \in X))$. Let $Y=X / G$ be the space of $G$-orbits, with the quotient topology; let $\pi_{0}: X \rightarrow Y$ be the canonical projection. Let $\gamma$ be normalized Haar measure on $G$, and fix a Radon measure $\nu_{0}$ on $Y$. Let $M^{\infty}\left(Y, \nu_{0}\right)$ be the algebra of all bounded $\nu_{0}$-measurable complex functions on $Y$, and let $L^{\infty}\left(Y, \nu_{0}\right)$ be the (usual) space of equivalence classes in $M^{\infty}\left(Y, \nu_{0}\right)$.

Definition 2.2. The Haar lift $\mu$ of $\nu_{0}$ is defined as follows: $\mu(f)=\int_{V}\left(\int_{G} f(g \cdot x) d \gamma(g)\right) d \nu_{0}(y)$ for each $f \in C(X)$.

Definition 2.3. Let $\rho_{0}$ be a fixed strong lifting ([6], 1.4; see the references given there) of $M^{\infty}\left(Y, \nu_{0}\right)$. Let $\rho$ be a linear lifting of $M^{\infty}(X, \mu)$. Note that $M^{\infty}\left(Y, \nu_{0}\right)$ may be embedded in $M^{\infty}(X, \mu)$ via $f \rightarrow f \circ \pi$. Say $\rho$ extends $\rho_{0}$ if $\left.\rho\right|_{M^{\infty}\left(Y, \nu_{0}\right)}=\rho_{0}$. Say $\rho$ commutes with $G$ if

$$
\rho(f \cdot g)(x)=\rho(f)(g \cdot x)\left(g \in G, x \in X, f \in M^{\infty}(X, \mu)\right) ;
$$

here $(f \cdot g)(x) \equiv f(g \cdot x)$.

The following theorem was proved in ([6]) subject to various additional assumptions. We prove it here in full generality.

THEOREM 2.4. Suppose $(G, X)$ is a free left transformation group. Let $\rho_{0}$ be a strong lifting of $M^{\infty}\left(Y, \nu_{0}\right)$. Then there exists a strong lifting $\rho$ of $M^{\infty}(X, \mu)$ which extends $\rho_{0}$ and commutes with 
$G$, where $\mu$ is the Haar lift of $\nu_{0}$.

More notation is necessary before we can discuss the proof of 2.4 .

Notation 2.5. Let $H$ be a closed, normal, real Lie subgroup of $G$. Let $Z=X / H$, and let $\pi: X \rightarrow Z$ be the projection. Note $(G / H, Z)$ is a free left transformation group. Write $g \cdot z$ for $(g H) \cdot$ $z(g \in G, z \in Z)$. Define a Radon measure $\nu$ on $Z$ by $\nu=\pi(\mu)$. Let $\lambda$ be normalized Haar measure on $H$. For each $z \in Z$, let $\lambda_{z}$ be the Radon measure on $X$ defined by $\lambda_{z}(f)=\int_{H} f(h \cdot x) d \lambda(h)$ for one (hence all) $x \in \pi^{-1}(z)$. Then $\mu(f)=\int_{Z} \lambda_{z}(f) d \nu(z)$ for all $f \in C(X)$.

It can be shown that 2.4 follows from 2.6 below. See the paragraphs under "Proof of 2.2, using 2.7" in ([6]), and the reference given there. See also the proofs of Theorems 2 and 3 in ([5], Chpt. IV).

THEOREM 2.6. Let $H, Z, \nu, \pi$ be as in 2.5, and suppose there is a strong lifting $\delta$ of $M^{\infty}(Z, \nu)$ which commutes with $G / H$. Then there is a strong lifting $\rho$ of $M^{\infty}(X, \mu)$ which extends $\delta$ and commutes with G.

To prove 2.6, we need only revise the proof of Proposition 3.11 in ([6]). For each $z_{0} \in Z$ and $f \in M^{\infty}(X, \mu)$, define $R^{f}\left(z_{0}\right)$ as in ([6], 3.3-3.5). Thus $R^{f}\left(z_{0}\right)$ is an element of $L^{\infty}\left(X, \lambda_{z_{0}}\right)$. Abusing notation, we think of $R^{f}\left(z_{0}\right)$ as a function on $\pi^{-1}\left(z_{0}\right)$. We repeat Proposition 3.9 of ([6]):

Proposition 2.7. $R^{f \cdot g}\left(z_{0}\right)\left(h \cdot x_{0}\right)=R^{f}\left(g \cdot z_{0}\right)\left(g h g^{-1} \cdot g z_{0}\right)\left(x_{0} \in X, z_{0}=\right.$ $\left.\pi\left(x_{0}\right), h \in H, g \in G\right)$.

Definition 2.8. Let $\left(V_{n}\right)_{n=1}^{\infty}$ be the $D^{\prime}$-sequence of $\S 1$. Let $x_{0} \in X, z_{0}=\pi\left(x_{0}\right)$. As in ([6], 3.10, Case I), define

$$
\begin{aligned}
T_{n}^{f}\left(x_{0}\right) & =\frac{1}{\lambda\left(V_{n}\right)} \int_{X} R^{f}\left(z_{0}\right)(\bar{x}) \psi_{V_{V_{n}} \cdot x_{0}}(\bar{x}) d \lambda_{z_{0}}(\bar{x}) \\
& =\frac{1}{\lambda\left(V_{n}\right)} \int_{H} R^{f}\left(z_{0}\right)\left(h x_{0}\right) \psi_{V_{n}}(h) d \lambda(h)
\end{aligned}
$$

(here $\psi$ denotes characteristic function).

Proposition 2.9. $T_{n}^{f . g}\left(x_{0}\right)=T_{n}^{f}\left(g \cdot x_{0}\right)\left(g \in G, x_{0} \in X\right)$. 
Proof.

$$
\begin{aligned}
& T_{n}^{f \cdot g}\left(x_{0}\right)=\frac{1}{\lambda\left(V_{n}\right)} \int_{H} R^{f \cdot g}\left(z_{0}\right)\left(h \cdot x_{0}\right) \psi_{V_{n}}(h) d \lambda(h) \\
& \quad=(\text { by } 2.7 \text { above }) \frac{1}{\lambda\left(V_{n}\right)} \int_{H} R^{f}\left(g \cdot z_{0}\right)\left(g h g_{-}^{-1} \cdot g x_{0}\right) \psi_{V_{n}}(h) d \lambda(h) \\
& \quad=(\text { by }([2], 28.72 \mathrm{e})) \frac{1}{\lambda\left(V_{n}\right)} \int_{H} R^{f}\left(g \cdot z_{0}\right)\left(h \cdot g x_{0}\right) \psi_{g V_{n} g^{-1}}(h) d \lambda(h) \\
& =T_{n}^{f}\left(g \cdot x_{0}\right) .
\end{aligned}
$$

2.10. Proof of 2.6. Combine the following: (i) the just-proved 2.9 ; (ii) the reasoning of the Case I portions of ([6], 3.12, 3.13, and 3.14); (iii) ([6], 3.15).

\section{REFERENCES}

1. R. Ewards and E. Hewitt, Pointwise limits for sequences of convolution operators, Acta Math., 113 (1965), 181-218.

2. E. Hewitt and K. Ross, Abstract Harmonic Analysis, Vol. II, Springer-Verlag, New York, Heidelberg, Berlin, 1970.

3. A. Ionescu-Tulcea, On the lifting property ( $V)$, Annals of Math. Stat., 36 (1965), 819-828.

4. A. and C. Ionescu-Tulcea, On the existence of a lifting ... locally compact group, Proc. Fifth Berk. Symp. Math. Stat. and Prob., Vol. 2, part 1, pp. 63-97.

5. — Topics in the Theory of Lifting, Springer-Verlag, New York, 1969.

6. R. Johnson, Existence of a strong lifting commuting with a compact group of transformations, to appear in Pacific J. Math.

7. A.A. Kirillov, Elements of the Theory of Representations, translated by Edwin Hewitt, Springer-Verlag, Berlin, Heidelberg, New York, 1976.

8. D. Montgomery and L. Zippin, Topological Transformation Groups, Interscience, New York, 1955.

9. A. Sagle and R. Walde, Introduction to Lie Groups and Lie Algebras, Pure and Applied Mathematics Series, Academic Press, New York, 1973.

Received July 13, 1978 and in revised form January 11, 1979.

UNIVERSity of SOUTHerN CALIFORNIA

Los ANGELes, CA 90007 
